\title{
Genetic diversity and structure in hill rice (Oryza sativa L.) landraces from the North- Eastern Himalayas of India
}

\author{
Somnath Roy ${ }^{1,5^{*}}$, B. C. Marndi ${ }^{2}$, B. Mawkhlieng ${ }^{1}$, A. Banerjee ${ }^{3,5}$, R. M. Yadav ${ }^{1}$, A. K. Misra ${ }^{1}$ and K. C. Bansal ${ }^{4}$
}

\begin{abstract}
Background: Hill rices (Oryza sativa L.) are direct seeded rices grown on hill slopes of different gradients. These landraces have evolved under rainfed and harsh environmental conditions and may possess genes governing adaptation traits such as tolerance to cold and moisture stress. In this study, 64 hill rice landraces were collected from the state of Arunachal Pradesh of North-Eastern region of India, and assessed by agro-morphological variability and microsatellite markers polymorphism. Our aim was to use phenotypic and genetic diversity data to understand the basis of farmers' classification of hill rice landraces into two groups: umte and tening. Another goal was to understand the genetic differentiation of hill rices into Indica or japonica subspecies.

Results: According to farmers' classification, hill rices were categorized into two groups: umte (large-grained, late maturing) and tening (small-grained, early maturing). We did not find significant difference in days to $50 \%$ flowering between the groups. Principal component analysis revealed that two groups can be distinguished on the basis of kernel length-to-width ration (KLW), kernel length $(K L)$, grain length $(G r L)$, grain length-to-width ration $(\mathrm{GrLW})$ and plant height $(\mathrm{Ht})$. Stepwise canonical discriminant analysis identified $\mathrm{KL}$ and $\mathrm{Ht}$ as the main discriminatory characters between the cultivar groups. Genetic diversity analysis with 35 SSR markers revealed considerable genetic diversity in the hill rice germplasm (gene diversity: 0.66; polymorphism information content: 0.62). Pair-wise allelic difference between umte and tening groups was not statistically significant. The model-based population structure analysis showed that the hill rices were clustered into two broad groups corresponding to Indica and Japonica. The geographic distribution and cultivars grouping of hill rices were not congruent in genetic clusters. Both distance- and model-based approaches indicated that the hill rices were predominantly japonica or admixture among the groups within the subspecies. These findings were further supported by combined analysis hill rices with 150 reference rice accessions representing major genetic groups of rice.
\end{abstract}

Conclusion: This study collected a valuable set of hill rice germplasm for rice breeding and for evolutionary studies. It also generated a new set of information on genetic and phenotypic diversity of hill rice landraces in North-Eastern region of India. The collected hill rices were mostly japonica or admixture among the subpopulations of Indica or Japonica. The findings are useful for utilization and conservation of hill rice germplasm.

Keywords: Rice (Oryza sativa), Diversity, Landrace, Hill rice, Exploration, Northeast India

\footnotetext{
* Correspondence: somnath.roy@icar.gov.in

${ }^{1}$ ICAR-National Bureau of Plant Genetic Resources, Regional Station, Umiam,

Meghalaya 793103, India

${ }^{5}$ Present address: ICAR-National Rice Research Institute, Cuttack, Odisha

753006, India

Full list of author information is available at the end of the article
} 


\section{Background}

Rice is the primary food source for more than half of the world's population [1]. Over $90 \%$ of the world's rice is produced and consumed in the Asia-Pacific region. Asian cultivated rice (Oryza sativa L.) is one of the important crops in the world. Being a model organism with fully sequenced genome, it also affords unique opportunities to use genomic approaches to study its domestication, adaptive selection, and the history of crop improvement $[2,3]$. The genetic structure of rice on a global scale is well characterized (see reviews in [4-6]). In addition to the two widely accepted major subspecies, Indica and Japonica, three to seven genetically distinct groups have been identified within global rice (Oryza sativa) germplasm sets in different studies [7-14]. Numerous studies on genetic structure of rice cultivars at a local scale (within a country) have also been conducted [15-22]. Such local-scale studies not only provide a detail view of rice genetic diversity within a country, but also allow for a better understanding of complex interaction between rice genetic diversity and human cultivation practices [18], and for formulating in situ conservation strategies [17]. The cultivated rice genetic diversity includes indigenous landraces and commercially bred improved varieties. As a consequence of both natural and artificial selection under diverse habitats, high levels of morphological, physiological and genetic diversity exist within O. sativa, resulting in $>120,000$ distinct rice varieties $[1,23]$. An assessment of this diversity is important for making decision about the conservation and effective utilization in breeding programmes.

The North-Eastern (NE) region of India, due to its unique eco-geographical features and high ethnic diversity, possesses at least 10,000 indigenous rice landraces which cultivated under upland, lowland and deep water conditions [24]. The impact of Green Revolution on the varietal landscape of rice in this region was insignificant, particularly in the hilly states, and the farmers still grow their heirloom varieties which not only suit their taste but also provide crop security. The current study estimates the phenotypic and genetic diversity present in a set of hill rice landraces collected from temperate to sub-temperate mountain region of the state of Arunachal Pradesh. The state of Arunachal Pradesh is situated on the eastern most corner of India sharing international borders with Bhutan, China and Myanmar. It has a geographical area of $83,743 \mathrm{sq}$. $\mathrm{km}$. The state has a population density of 17 persons per sq. $\mathrm{km}$, and around $68.8 \%$ of the population is tribal [25]. The tribal communities of Arunachal Pradesh are of Tibeto-Burman linguistic origin, and there are as many as 21 tribes and 50 sub-tribes. The topography of the state is mostly hilly terrain and the agroclimate varies from tropical to alpine. The farmers follow traditional shifting or swidden cultivation (locally known as jhum) and sedentary agriculture. Shifting or 'slash-and-burn' cultivation is the earliest form of agriculture and is still practiced in vast areas of the state. About $76 \%$ of the total cropped area in the state is under jhum cultivation.

Rice is the principal crop of Arunachal Pradesh and it is grown in $44.7 \%$ of the total cropped land and accounts for $70.3 \%$ of the total food grain production [25]. Rice productivity in the state is very low $(2065 \mathrm{~kg} / \mathrm{ha})$. Here rice is grown up to an altitude of $2000 \mathrm{~m}$ as a rainfed crop in hill slopes of newly cleared forests (jhum fields), plateaus, terraces and river beds. A wide range of rice landraces were found throughout the state. Sometimes it depends on the tribal communities who inhabit the area. It was estimated that more than $70 \%$ of the total rice varieties grown in the state are traditional cultivars or landraces having low to very low productivity [26]. Despite having low yield potential, the hill rice landraces grown in the mountains under jhum cultivation system possess many adaptive traits such as cold tolerance important for crop improvement programmes. Cold-tolerance in hill rice landraces was also observed in high altitude (13002200 m) rice areas in Nepal [27]. Similarly, in the high altitude rice areas of Arunachal Pradesh cold injury due to cool air temperature, particularly during flowering stage, is a major constraint to improving rice productivity. In general, hill rice landraces are grown on the cleared mountain sides for one year and then left fallow for 4-5 years before the farmers return to the same location (Additional file 1). The farmers usually grow few maize and millets along with rice in the same field. The hill rice landraces have been evolved by the interplay between adaptation to the harsh environment and selection imposed by the farmers who determine which varieties will be grown under a particular agro-ecological condition. Therefore, it will be interesting to study the impact of farmers' practices and local culture on the conservation, exchange and genetic structure of traditional landrace varieties, by studying rice landraces collected from a unique ecological niche like mountain sides. There is no previous study done on genetic diversity of the hill (jhum) rice cultivars of Arunachal Pradesh.

In 2012, an exploration trip to three districts viz, East Kameng, Papum Pare and Kurung Kumey of the state of Arunachal Pradesh was conducted for the collection of hill rice landraces. These rices are direct seeded, grown on upland fields, usually on sloping hill sides (jhum lands), and are grown as rainfed during June to September. During the collection trip, we interviewed local farmers to gather information on significance of naming a landrace, its cultural value and popularity, and production practices of local farmers. The indigenous farmers of the surveyed region rely on gathering forest products, hunting and cultivation of rice and maize for their 
subsistence. In the present study, we phenotyped these rice landraces for 16 agro-morphological and grain characteristics, and genotyped them with 35 SSR markers. The specific objectives of this study were to: (i) collect information on the meanings of the variety names, special uses or properties, and important agronomic characteristics; (ii) study the agro-morphological variability; and (iii) understand the genetic relatedness and structure of these landraces.

\section{Methods}

\section{Collection of hill rice}

A total of 67 hill rice accessions were collected from 32 villages in three districts (East Kameng, Papum Pare and Kurung Kumey) of Arunachal Pradesh, India during a collection trip from November 2-15, 2012 (Additional file 2). The geographical distribution of collected landraces is depicted in Fig. 1. The collection team, consisting of two researchers (SR and $\mathrm{BCM}$ ) and local guide from Krishi Vigyan Kendras (Farm Science Centres) surveyed the districts and collected the landraces. In each village, seed samples were taken from the freshly harvested seed lots conserved in the farm stores. However, in some villages of Papum Pare, the seeds were collected from the standing crop. Due to poor road connectivity, the team was unable to explore very remote villages. Although the elevation of the collection sites varied from $135 \mathrm{~m}$ (Seijosa, East Kameng) to $1425 \mathrm{~m}$ (Sarli in Kurung Kumey), but the actual rice fields were much higher. Rice seeds were mostly collected from the households situated in the foothills or in the valleys. During collection, the farmers were interviewed to acquire information about the specific varieties: the meaning of cultivar name, source or origin, grain quality and special uses, disease and pest resistance, and yield.

\section{Plant materials}

Out of these 67 hill rice accessions, altogether 64 were selected for agro-morphological and genetic characterization in this study. Information about the accessions such as name, accession number, place of collection/seed sources, farmers' grouping of landraces and subpopulation ancestry based on STRUCTURE is given in Additional file 3 . The seeds of the hill rice accessions used in this study are conserved in the National Genebank of ICAR-National Bureau of Plant Genetic Resources (NBPGR), New Delhi, India, and are publicly available for research purposes upon request with a material transfer agreement. The rice accessions were grown in 2013 and 2014 for purification. In each year, seeds from a single panicle were kept for sowing in the next year.

Three check varieties such as 'Bhalum-1', 'Bhalum-3' and 'Sukhadhan-1' were used during phenotypic characterization trial. 'Bhalum-1' and 'Bhalum-3' varieties are suited for cultivation under rainfed upland conditions up to an altitude of $1000 \mathrm{~m}$. 'Sukhadhan-1' is a drought tolerant upland rice variety of Nepal. Seed of these accessions were obtained from ICAR Research Complex for North-Eastern Hill Region, Umiam, India.

A total of 15 control varieties were selected for SSR genotyping along with the hill rices (Additional file 3). These checks included five indica ('IR8', 'IR36', 'Jaya', 'Taichung Native 1' and 'TKM9'), two aus ('FR 13A' and 'Kalamkati'), three Basmati ('Basmati370', 'Pakistani Basmati' and 'Pusa Basmati 1'), three short-grain

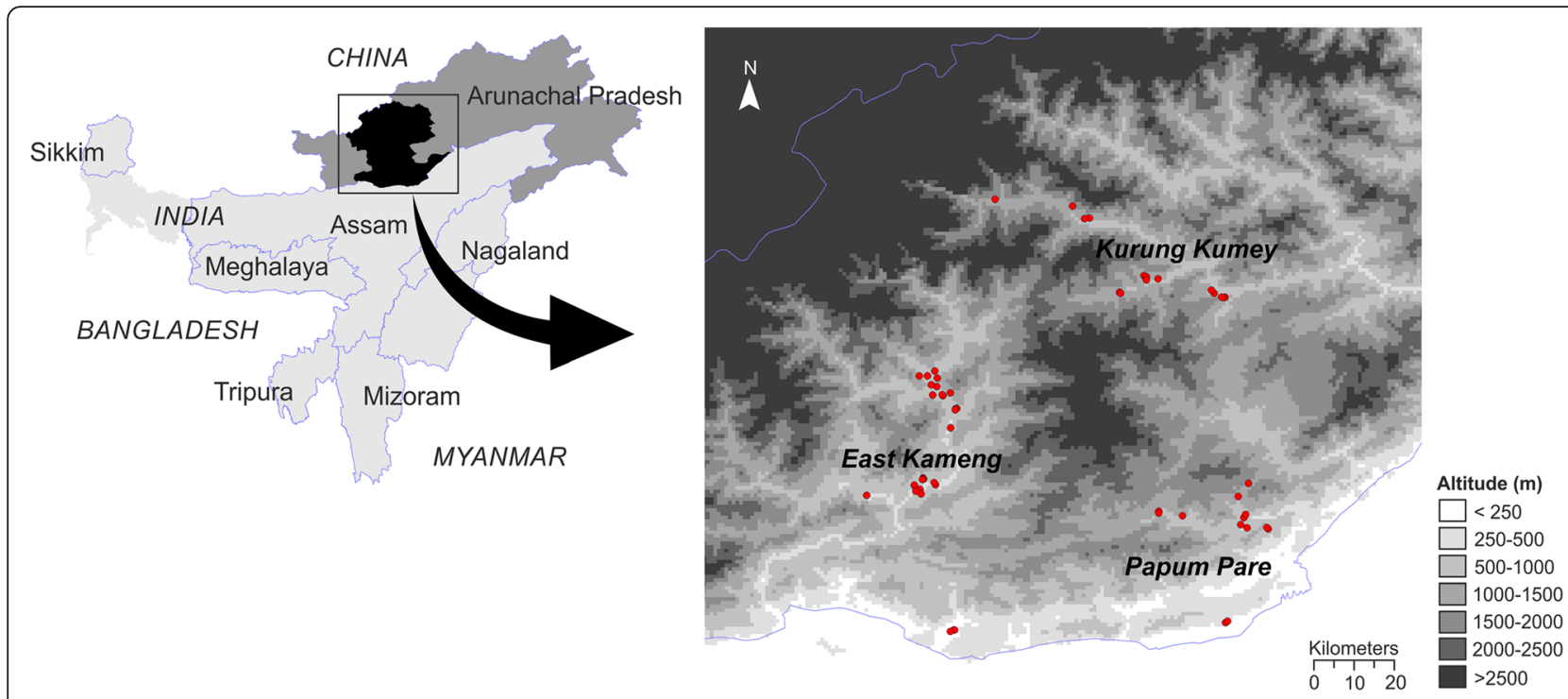

Fig. 1 Geographic locations of the hill rice collection sites in three districts of the state of Arunachal Pradesh 
traditional aromatic ('Badshabhog', 'Kataribhog' and 'Mohanbhog') and a single accession each of tropical japonica ('Azucena') and japonica admixture ('Dular'). Pure seeds of these accessions were obtained from ICAR-National Rice Research Institute, Cuttack, India. These varieties were used to understand genetic grouping of the hill rice accessions, and also to act as a molecular weight reference in DNA gel electrophoresis. We did not include these varieties in phenotypic evaluation as the plant growth was very poor for most of the accessions.

To provide a reference for the rice subpopulation classification in the current study, we also used the SSR fingerprinting data of 150 rice accessions (here we denoted these as reference accessions) previously reported in the genetic diversity study by Garris et al. [9]. The band size data were obtained from 'Gramene genetic diversity database' (http://archive.gramene.org/db/diversity/diversity_view). We sampled 150 accessions from the originally reported 234 accessions used in Garris et al. [9] based on the recent report on open access resources for genome-wide association mapping in rice [28].

\section{Agro-morphological characterization}

Sixty four hill rice accessions and three check varieties ('Bhalum-1', 'Bhalum-3' and 'Sukhadhan-1' were grown at experimental field of NBPGR, Regional Station, Umiam, Meghalaya $\left(25.6^{\circ} \mathrm{N}\right.$ lat, $91.9^{\circ} \mathrm{E}$ long and $1000 \mathrm{~m}$ alt) in 2015 Kharif season (June-November) under rainfed upland conditions. The soil of the field is an acid alfisol with a pH of 5.3, organic C $1.13 \%$ and available $\mathrm{N}$ $211 \mathrm{~kg} / \mathrm{ha}$. Farmyard manure (10 tonnes/ha) was incorporated into the soil during land preparation. No chemical fertilizers and pesticides were used during crop growth period. A manual weeding was conducted to keep the crop weed free. The accessions were line-sown in $1 \mathrm{~m} \mathrm{x} 1.5 \mathrm{~m}$ plots (4 lines/accession) using an augmented block design during first week of June. A spacing of $\sim 0.25 \mathrm{~m}$ between the plants and $0.45 \mathrm{~m}$ between the rows was maintained. Phenotypic data, listed in Table 1, were recorded following the rice descriptors used by International Rice Research Institute [29]. Days required from sowing to $50 \%$ flowering (DtF) was recorded on plot basis. The traits such as plant height $(\mathrm{Ht})$, leaf length (LL), leaf width (LW), ligule length $(\mathrm{LgL})$, panicles per plant $(\mathrm{PnP})$ and ligule length (LgL) were recorded as the average of five randomly selected plants. Panicle length $(\mathrm{PnL})$ and spikelets per panicle $(\mathrm{SnP})$ were measured based on five individual measurements on the main stem. Grain and kernel morphological traits were determined from randomly sampled ten grains/kernels. The 1000-grain weight (GW) was calculated by taking weight of 100 grains from the bulk and multiplying it with 10 .

\section{Genotyping with SSR markers}

Thirty five SSR markers were designed from the 'Gramene' marker database (http://www.gramene.org/ markers/microsat/). Information about these markers is given in Additional file 4. Extraction of total genomic DNA, polymerase chain reaction (PCR) with SSR markers and molecular size determination of amplified band were performed as described in Roy et al. [30]. During gel electrophoresis at least one control accession of 'IR8', 'IR36', 'Taichung Native 1', 'FR 13A', 'Kalamkati' or 'Dular' was used as a molecular weight reference in each gel because a reference allele size in these control accessions is available [9]. The original SSR genotype data of all rice accessions used in this study are provided in Additional file 5.

\section{Agro-morphological data analysis}

Summary statistics such as mean, standard deviation (SD), minimum and maximum values, and coefficient of variation $(\mathrm{CV})$ were determined. The mean data were standardized by deriving $Z$ scores for further analyses. ANOVA was carried out to test the significance of variation between hill rice cultivar groups for different traits. Principal component analysis (PCA) was performed on the correlation matrix of the data to understand the most important variables contributing to the total phenotypic variation among the accessions. PCA represents the simplest and most commonly used multivariate method to visualize the grouping of accessions based on component loadings. Ward's hierarchical clustering was used to understand the relationships among rice accessions based on phenotypic data. All these analyses were performed using IBM-SPSS Statistics version 20.0 [31].

\section{Population structure analysis}

Population structure of 64 hill and 15 control rice accessions was examined using the Bayesian model-based approach implemented in STRUCTURE V2.3.4 [32]. The number of clusters $(K)$ evaluated here ranged from 1 to 8. The analysis was performed using five replicate runs per $K$ value, a burn-in period length of 5000, a run length of 50,000, and a model allowing for admixture and correlated allele frequency. 'Structure harvester' programme (http://taylor0.biology.ucla.edu) was used to determine the final $K$ value(s) based on both the $\operatorname{LnP}(\mathrm{D})$ and Evanno's $\Delta K$ [33]. Subsequently, ten simulations at $K=2-4$ were then performed with a burn-in period of 10,000 and a run length of 100,000. The membership coefficient from the run with the lowest likelihood value was used to assign each accession to the $K=1$ to 4 subpopulations based on the estimated membership coefficients.

\section{Summary statistics and genetic diversity analysis}

The average number of alleles per locus (AN), major allele frequency (MAF), gene diversity $\left(H_{e}\right)$, heterozygosity 
Table 1 Range of variation in agro-morphological characteristics of hill rice landraces and the loading of the measured traits on five principal components

\begin{tabular}{|c|c|c|c|c|c|c|c|c|c|c|c|}
\hline \multirow[t]{2}{*}{ Traits } & \multirow[t]{2}{*}{ Group } & \multirow[t]{2}{*}{ Mean \pm SD } & \multirow[t]{2}{*}{ Min } & \multirow[t]{2}{*}{ Max } & \multirow[t]{2}{*}{$\% C V$} & \multirow[t]{2}{*}{$p$ value } & \multicolumn{5}{|l|}{ PCs } \\
\hline & & & & & & & 1 & 2 & 3 & 4 & 5 \\
\hline \multirow[t]{2}{*}{$\overline{\mathrm{DtF}}$} & Umte & $115.78 \pm 13.05$ & 90.00 & 141.00 & 11.27 & 0.110 & -0.56 & 0.31 & 0.48 & -0.38 & -0.04 \\
\hline & Tening & $120.84 \pm 11.79$ & 89.00 & 139.00 & 9.75 & & & & & & \\
\hline \multirow[t]{2}{*}{$\mathrm{Ht}$} & Umte & $81.11 \pm 23.52$ & 32.00 & 122.20 & 29.00 & 0.034 & 0.58 & -0.39 & -0.48 & 0.20 & 0.04 \\
\hline & Tening & $69.86 \pm 17.90$ & 40.20 & 107.00 & 25.62 & & & & & & \\
\hline \multirow[t]{2}{*}{$\mathrm{LL}$} & Umte & $53.76 \pm 11.04$ & 36.00 & 75.00 & 20.53 & 0.053 & -0.60 & 0.34 & 0.21 & -0.40 & -0.16 \\
\hline & Tening & $59.23 \pm 10.87$ & 38.60 & 78.70 & 18.36 & & & & & & \\
\hline \multirow[t]{2}{*}{ LW } & Umte & $1.86 \pm 0.21$ & 1.40 & 2.30 & 11.22 & 0.270 & -0.49 & 0.30 & -0.24 & -0.08 & -0.39 \\
\hline & Tening & $1.92 \pm 0.25$ & 1.40 & 2.40 & 13.10 & & & & & & \\
\hline \multirow[t]{2}{*}{ LgL } & Umte & $1.65 \pm 0.53$ & 0.95 & 2.90 & 31.92 & 0.390 & 0.59 & -0.06 & 0.08 & -0.43 & 0.16 \\
\hline & Tening & $1.56 \pm 0.38$ & 0.70 & 2.35 & 24.56 & & & & & & \\
\hline \multirow[t]{2}{*}{$\mathrm{PnP}$} & Umte & $5.19 \pm 0.97$ & 3.50 & 7.20 & 18.76 & 0.410 & 0.11 & -0.01 & 0.66 & 0.38 & -0.29 \\
\hline & Tening & $5.39 \pm 0.97$ & 3.50 & 7.30 & 18.02 & & & & & & \\
\hline \multirow[t]{2}{*}{$P n L$} & Umte & $23.17 \pm 2.87$ & 16.50 & 27.75 & 12.38 & 0.149 & 0.44 & -0.46 & 0.30 & -0.09 & 0.48 \\
\hline & Tening & $22.16 \pm 2.65$ & 17.50 & 28.65 & 11.96 & & & & & & \\
\hline \multirow[t]{2}{*}{$\mathrm{SnP}$} & Umte & $143.07 \pm 52.53$ & 82.50 & 272.50 & 36.72 & 0.234 & -0.14 & -0.53 & 0.63 & 0.10 & 0.20 \\
\hline & Tening & $159.89 \pm 57.13$ & 68.00 & 260.50 & 35.73 & & & & & & \\
\hline \multirow[t]{2}{*}{ GrL } & Umte & $6.94 \pm 0.70$ & 5.27 & 8.31 & 10.05 & 0.000 & 0.58 & 0.68 & 0.14 & 0.01 & 0.11 \\
\hline & Tening & $6.19 \pm 0.65$ & 5.17 & 8.32 & 10.44 & & & & & & \\
\hline \multirow[t]{2}{*}{ GrW } & Umte & $3.39 \pm 0.37$ & 2.67 & 3.87 & 10.81 & 0.407 & -0.81 & 0.23 & 0.04 & 0.28 & 0.22 \\
\hline & Tening & $3.46 \pm 0.37$ & 2.73 & 4.01 & 10.58 & & & & & & \\
\hline \multirow[t]{2}{*}{ GrLW } & Umte & $2.07 \pm 0.31$ & 1.73 & 2.97 & 14.81 & 0.003 & 0.87 & 0.32 & 0.08 & -0.17 & -0.11 \\
\hline & Tening & $1.81 \pm 0.34$ & 1.40 & 2.91 & 18.87 & & & & & & \\
\hline \multirow[t]{2}{*}{ StLL } & Umte & $2.31 \pm 0.50$ & 1.46 & 3.29 & 21.48 & 0.240 & 0.07 & 0.42 & -0.15 & -0.43 & 0.60 \\
\hline & Tening & $2.19 \pm 0.30$ & 1.48 & 2.98 & 13.82 & & & & & & \\
\hline \multirow[t]{2}{*}{ GW } & Umte & $23.03 \pm 4.32$ & 15.80 & 34.60 & 18.77 & 0.843 & -0.25 & 0.49 & -0.06 & 0.58 & 0.38 \\
\hline & Tening & $22.85 \pm 3.09$ & 16.90 & 32.80 & 13.54 & & & & & & \\
\hline \multirow[t]{2}{*}{$\mathrm{KL}$} & Umte & $5.44 \pm 0.46$ & 4.48 & 6.24 & 8.36 & 0.000 & 0.70 & 0.49 & 0.16 & 0.34 & 0.04 \\
\hline & Tening & $4.58 \pm 0.42$ & 3.90 & 6.06 & 9.14 & & & & & & \\
\hline \multirow[t]{2}{*}{$\mathrm{KW}$} & Umte & $2.9 \pm 0.35$ & 2.12 & 3.45 & 12.12 & 0.133 & -0.84 & 0.11 & -0.04 & 0.17 & 0.26 \\
\hline & Tening & $3.02 \pm 0.24$ & 2.44 & 3.35 & 8.10 & & & & & & \\
\hline \multirow[t]{2}{*}{ KLW } & Umte & $1.91 \pm 0.34$ & 1.59 & 2.94 & 17.82 & 0.000 & 0.89 & 0.28 & 0.14 & 0.12 & -0.13 \\
\hline & Tening & $1.53 \pm 0.25$ & 1.23 & 2.48 & 16.11 & & & & & & \\
\hline Percentage Variance & & & & & & & 35.18 & 14.43 & 0.89 & 9.23 & 7.64 \\
\hline Cumulative variance & & & & & & & 35.18 & 49.61 & 59.50 & 68.73 & 76.3 \\
\hline
\end{tabular}

$D t F$ days to $50 \%$ flowering, $H t$ plant height $(\mathrm{cm}), L L$ leaf length $(\mathrm{cm}), L W$ leaf width $(\mathrm{cm}), L g L$ ligule length $(\mathrm{cm}), P n P$ panicles per plant, $P n L$ panicle length $(\mathrm{cm})$, SnP spikelets per panicle, GrL grain length $(\mathrm{mm}), G r W$ grain width $(\mathrm{mm}), \mathrm{GrLW}$ grain length-to-width ratio, StLL sterile lemma length $(\mathrm{mm}), G W$ 1000-grain weigth $(\mathrm{g})$, $K L$ kernel length $(\mathrm{mm}), K W$ kernel width $(\mathrm{mm}), K L W$ kernel length-to-width ratio, $S D$ standard deviation, Min minimum value, Max maximum value, $C V$ coefficient of variation, $P C$ principal component

$\left(H_{o}\right)$ and polymorphism information content (PIC) were calculated using PowerMarker V3.25 [34]. Average allelic richness $\left(R_{s}\right)$ and Wright's fixation index $\left(F_{s t}\right)$ values were calculated using FSTAT V2.9.3.2 [35]. The molecular variance of subpopulations and accessions within the subpopulations were calculated using an Analysis of
Molecular Variance (AMOVA) approach in GenAlEx V6.5 [36]. Separate analyses were conducted by classifying the rice accessions into: districts, farmers' classified groups and STRUCTURE subpopulations. The statistical test of the differences in allelic count among different groups with variable numbers of accessions was 
conducted in $\mathrm{R}$ [37] using FPTest $\mathrm{R}$, the $\mathrm{R}$ version of FPTest method reported in Fu et al. [38]. The FPTestR package (communicated for publication) was kindly provided by $\mathrm{Dr}$ Yong-Bi Fu, Plant Genetic Resources of Canada, Agriculture and Agri-Food Canada. The allele frequency data from PowerMarker was used to export the data in binary format (1/0) for analysis with NTSYS-pc V2.2 [39]. A neighbour-joining (NJ) cluster diagram was constructed with the NJOIN sub-programme using the genetic dissimilarity matrix calculated in SIMGEND subprogramme with Nei72 coefficient. To summarize the patterns of variation in multi-locus dataset, principal coordinate analysis $(\mathrm{PCoA})$ was performed in GenAlex software using the genetic distance matrix among the accessions.

\section{Combined analysis of hill and reference accessions}

Cluster analysis was conducted on hill and reference accessions to understand the grouping of hill rices in respect to different genetically defined groups (indica, aus, aromatic, tropical japonica and temperate japonica). The reference set also included some 'admixture' accessions. For clustering, a pair-wise genetic distance matrix was calculated in PowerMarker following C. S. Chord distance method [40]. The NJ method was used for phylogenetic reconstruction. The unrooted NJ tree was visualized using Dendroscope V3 [41]. Pair-wise $F_{s t}$ and allelic differences (FPTest) between the groups were calculated as stated earlier.

\section{Results}

\section{Variability in agro-morphological traits}

Based on farmers' classification of the hill rices, the collected rice germplasm was categorized into two cultivar types: (i) umte (um=rice; te=large), large grained-late maturing rice, and (ii) tening, small grained-early maturing types (Additional file 3). The umte cultivars are high yielding and good to taste. In this study, we have assessed the agro-morphological and genetic diversity between these two groups.

Descriptive statistics for 16 agro-morphological characteristics in umte and tening groups of hill rice are given in Table 1 . The variation observed between two groups was significant for the traits evaluated such as plant height $(\mathrm{Ht})$, leaf length (LL), grain length $(\mathrm{GrL})$, grain length-to-width ratio (GrLW), kernel length (KL) and kernel length-to-width ratio (KLW). There was no significant difference in days to $50 \%$ flowering (DtF) between gropus. The range of variation in DtF in both the groups was similar, and interestingly tening group showed higher average value of DtF (120.8 d) than umte $(115.8 \mathrm{~d})$. However, the difference was prominent for grain and kernel characteristics. The umte accessions had longer grains with an average value of $6.9 \mathrm{~mm}$ than that of tening $(6.2 \mathrm{~mm})$. Similarly, the value of $\mathrm{KL}$ in umte $(5.4 \mathrm{~mm})$ was higher than that recorded in tening $(4.6 \mathrm{~mm})$. Both the highest values for GrLW and KLW were recorded in umte. On average, the plants of umte accessions $(81.1 \mathrm{~cm})$ were taller than the tenings $(69.9 \mathrm{~cm})$. The tenning accessions produced larger leaves $(59.7 \mathrm{~cm})$.

Principal component analysis (PCA) using 16 traits produced five principal components (Eigenvalue $>1$ ) which cumulatively accounted for $76.4 \%$ of the total phenotypic variance (Table 1). The first PC explained $35.2 \%$ of the total variance and the traits with high positive loadings were KLW, GrLW, KL, GrL, LgL (ligule length) and $\mathrm{Ht}$. The PC2 explained an additional $14.4 \%$ of the total variance. The traits such as GrL, KL and 1000-grain weight (GW) showed high positive loadings. The variation in panicles per plant $(\mathrm{PnP})$, spikelets per panicle $(\mathrm{SnP})$ and $\mathrm{DtF}$ was largely explained by $\mathrm{PC} 3$ (Table 1). The PCA biplot with PC1 and PC2 scores showed that the accessions belonging to umte and tening groups clustered separately based on the phenotypic traits (Fig. 2a). The umte accessions having higher values for GrL, GrLW, KL, KLW, Ht and PnL, positioned at the right half of the biplot. The tening accessions occupied the left half of biplot mainly because of higher values for the traits such as SnP, GrW and KW. The three check varieties used in phenotypic characterization were grouped separately from most of the hill rice accessions.

Ward's hierarchical cluster analysis revealed a similar grouping of accessions as found in PCA (Fig. 2b). Overall, 67 accessions were divided into two groups. All check varieties formed a distinct cluster $(\mathrm{C} 1)$ along with ten hill accessions. The $\mathrm{C} 2$ was further divided into two sub-clusters. There was no clear grouping of umte and tening accessions, except a sub-group (C2B) of 15 tenning accessions which had highest values for KW, GrLW, DtF, LL and leaf width (LW).

\section{SSR variation}

The summary statistics of 35 SSR markers are given in Additional file 4. Overall, 297 alleles were detected at the 35 SSR markers, ranging from 2 alleles (RM338) up to 21 alleles (RM259), with an average of 8.49 alleles across the loci. The gene diversity or expected heterozygosity $\left(H_{e}\right)$ ranged from 0.41 (RM55) to 0.94 (RM259) and the average observed heterozygosity $\left(H_{o}\right)$ was 0.051 . Allelic richness $\left(R_{s}\right)$ varied from 2.0 (RM338) to 14.6 (RM259) with an average value of 6.04 across the study. The polymorphism information content (PIC) values ranged from 0.37 (RM338 and RM507) up to 0.93 (RM259) with an average of 0.65 .

\section{Genetic structure and subdivision of hill rice accessions}

The inferred population structures are given in Fig. 3a and Additional file 3. By comparing $\operatorname{LnP}(\mathrm{D})$ and Evanno's 
a

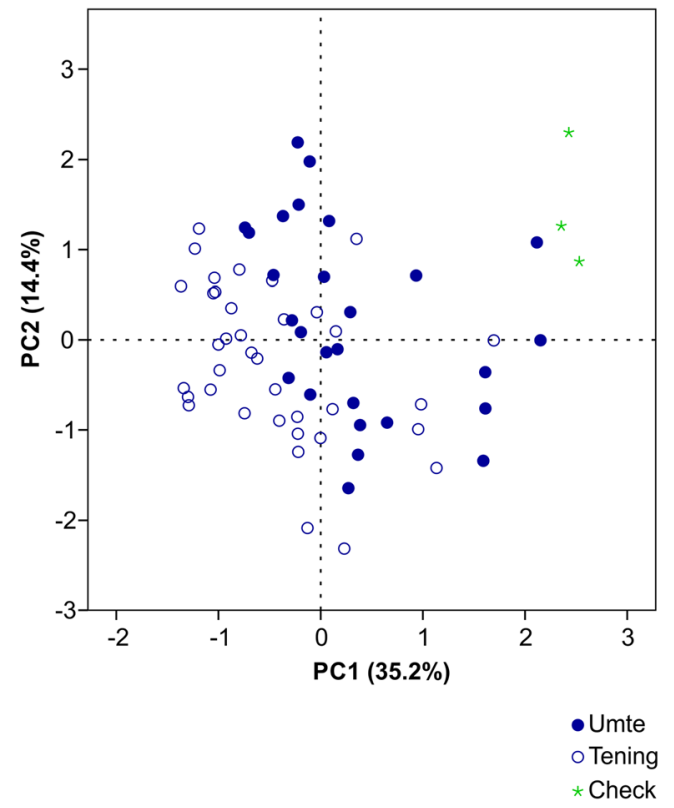

b

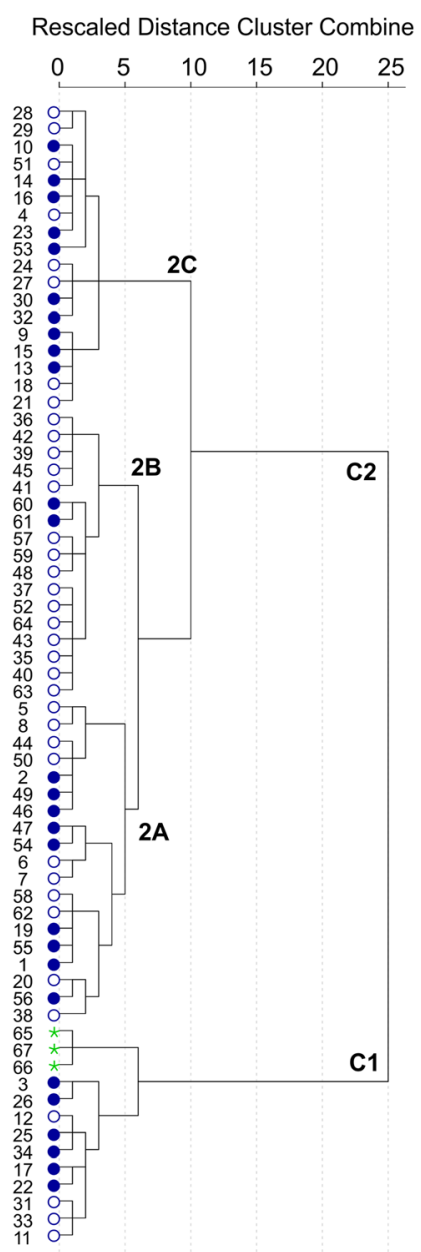

Fig. 2 Grouping of the hill rice landraces based on 16 agro-morphological characteristics. a Principal component bioplot constructed using first two principal components; $\mathbf{b}$ Ward's hierarchical cluster clustering. The serial numbers of rice accessions are given in Additional file 3. Umte and tening accessions are represented using filled and hollow circles, respectively

$\Delta K$ values by increasing $K$ from 1 to 8 , we found that $\mathrm{LnP}(\mathrm{D})$ values increased with $K$, with the highest log likelihood score at $K=2$, while $\Delta K$ value was also highest at $K=2$ (Additional file 6). No peak of $\Delta K$ was evident at $K>3$. This indicated that these 64 hill and 15 control accessions had a genetic structure of two subpopulations, supporting occurrence of two subspecies: Indica and Japonica. However, we have presented the structures at $K=2$ to 4 , to check for biological relevance. At $K=2$, group 1 had 32 accessions including 11 control varieties belonging to indica, aus and aromatic. Altogether, 10 admixture accessions (having $<80 \%$ of inferred ancestry from any one group) including 'Azucena', 'TKM9' and 'Pakistani Basmati' were identified in this group. Group 2 consisted of a total of 47 accessions including 'Dular', a japonica admixture variety, as admixture. At $K=3$, ten control varieties (indica, aus and aromatic) formed group 1 along with 11 hill accessions. Group 2 has
15 hill rices along with 'Dular' as admixture. A majority of hill rices (37 accessions) were included in group 3 along with the control varieties such as 'Azucena', 'TKM9', 'Pakistani Basmati' and 'Mohanbhog'. The subpopulation structure in these rice accessions was more clear at $K=4$. The four subpopulations (StrGr1 to 4) had a populationspecific $F_{s t}$ value of $0.33,0.33,0.06$ and 0.20 , respectively, with an average of 0.23 , indicating a moderate population structure. At this level, indica control varieties were separated from aus and aromatic varieties. The aromatic tropical japonica variety 'Azucena' showed separate grouping along with $50 \%$ of hill accessions. A group of 15 hill accessions formed a distinct group (StrGr1). At $K=4$, a total of 25 accessions were identified as an admixture.

The neighbour-joining (NJ) analysis of pair-wise genetic distances detected four major clusters (C1-C4; Fig. 4a). These clusters showed varied regional heterogeneity. 


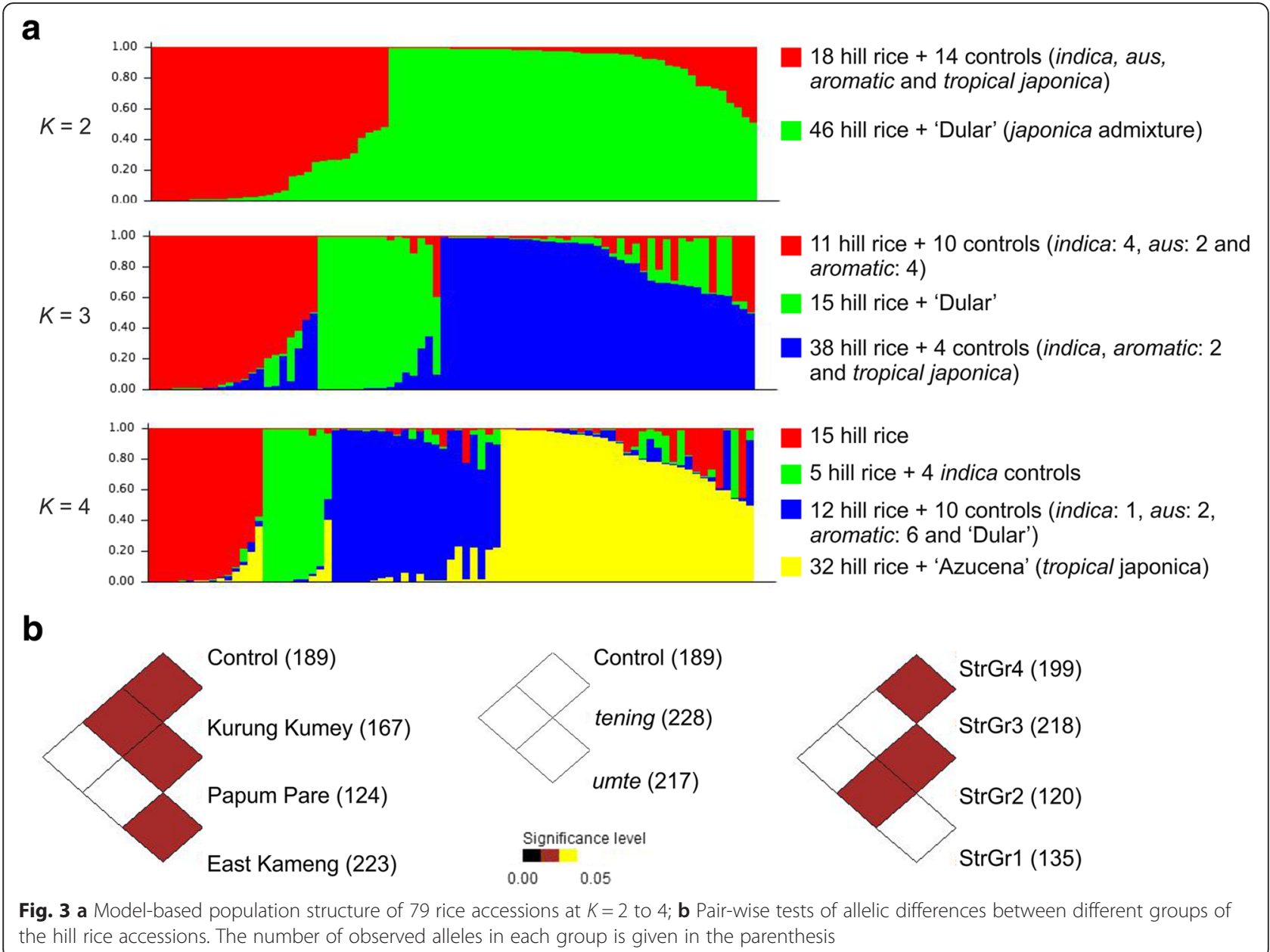

However, the hill accessions collected from Papum Pare district grouped in cluster 4 . Cluster 1 was consisted of all indica and aus controls, along with four hill accessions collected from Kurung Kumey and a single accession from East Kameng. Cluster 2 grouped all aromatic control varieties and four hill accessions collected from East Kameng. The rest hill rices grouped either with tropical japonica variety 'Azucena' (53 accessions) or with 'Dular' (3 accessions). The cluster membership of each accession is given in Additional file 3. Upon labelling the accessions according to their inferred STRUCTURE ancestry, we did not find exact match between the results of $\mathrm{NJ}$ cluster and STRUCTURE $(K=4)$. However, the PCoA results were consistent with STRUCTURE (Fig. 4b). In PCoA, first two coordinates clearly separated four STRUCTURE subpopulations. The indica, aus and aromatic control varieties formed a distinct group in the PCoA plot. Note that the farmers' classification of hill rices into umte and tening group based on morphological traits was not supported by any of the analyses. Taken together, all three approaches demonstrated that the majority of these hill rice accessions are Japonica or admixture among the groups within either Japonica or Indica.

\section{Genetic diversity}

The rice accessions displayed different pattern of SSR variation with respect to district, cultivar group and STRUCTURE grouping (Table 2). The AMOVA revealed significant variations $(p=0.001)$ among and within subpopulation groups corresponding to districts, STRUCTURE groups and overall hill and control accessions (Additional file 7). However, a non-significant $(p=0.089) \quad 0.8 \%$ of the total variation among umte and tening groups was recorded. Considering the districts, the highest genetic diversity was observed in East Kameng accessions (0.65) with a mean of 6.31 alleles per locus. The lowest genetic diversity was recorded in the accessions from Papum Pare (0.50) with a mean of 3.54 alleles per locus (Table 2). Between umte and tening groups, the genetic diversity and mean number of alleles per locus (AN) was identical. The umte group had the highest PIC value in spite of having lower number of accessions. Among four STRUCTURE subpopulations, 


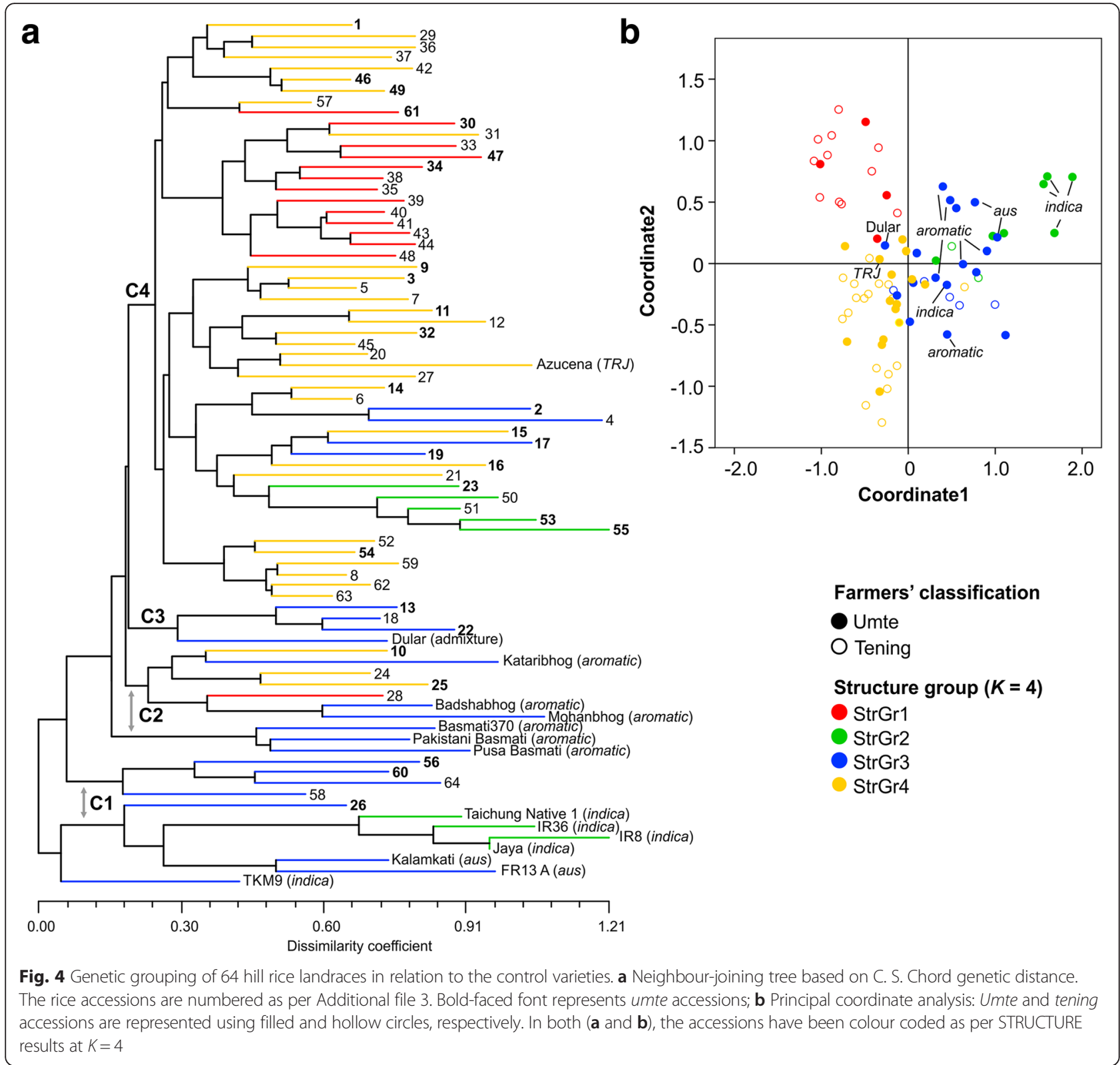

the highest genetic diversity was observed in StrGr3 (0.69) with an AN of 6.26, followed by StrGr4 (0.59) with an AN of 5.69. Similar trends were observed for PIC and allelic richness $\left(R_{s}\right)$ values. The overall genetic diversity in hill and control accessions was at par, but the hill rices had the highest $R_{s}$ (5.47) and AN (7.4). Note that, despite having only 15 accessions, the control group recorded the highest genetic diversity and PIC. This may be due to inclusion of diverse rice control varieties from different genetic groups.

The number of alleles in a sample (allelic count or allelic richness) is an important measure of diversity. Pair-wise tests of allelic differences between different groups of accessions are given in Fig. 3b. Allelic counts of Papum Pare and Kurung Kumey was significantly different with that of the control. The allelic differences between the districts were also significant, except the case between East Kameng and Kurung Kumey. No significant allelic differences were noted between umte and tening, or between controls and either of the two groups. Among the four STRUCTURE subpopulations, allelic count of StrGr3 was significantly different with that of StrGr1 and StrGr2 (Fig. 3b). Pair-wise allelic difference between StrGr3 and StrGr4 was also significant.

Pair-wise estimates of $F_{s t}$ values among and between subpopulations are given in Additional file 8. The level of differentiation between umte and tening was very low (0.008). Overall, the rice accessions from three districts 
Table $\mathbf{2}$ Genetic diversity statistics of hill rice landraces at different sub-population levels

\begin{tabular}{lllllll}
\hline Sub-population & $\mathrm{n}$ & $\mathrm{AN}$ & $\mathrm{MAF}$ & $R_{s}$ & $H_{e}$ & $\mathrm{PIC}$ \\
\hline Districts & & & & & & \\
East Kameng & 34 & 6.31 & 0.49 & 5.32 & 0.65 & 0.61 \\
Papum Pare & 13 & 3.54 & 0.62 & 3.51 & 0.50 & 0.45 \\
Kurung Kumey & 17 & 4.77 & 0.51 & 4.54 & 0.61 & 0.56 \\
Farmers' classified groups & & & & & & \\
Umte & 27 & 6.14 & 0.46 & 5.54 & 0.67 & 0.63 \\
Tening & 37 & 6.51 & 0.50 & 5.30 & 0.63 & 0.59 \\
STRUCTURE & & & & & & \\
sub-populations (K=4) & & & & & & \\
StrGr1 & 15 & 3.77 & 0.61 & 3.51 & 0.51 & 0.46 \\
StrGr2 & 9 & 3.49 & 0.56 & 3.49 & 0.53 & 0.48 \\
StrGr3 & 22 & 6.26 & 0.44 & 5.20 & 0.69 & 0.65 \\
StrGr4 & 33 & 5.69 & 0.54 & 4.42 & 0.59 & 0.55 \\
All hill accessions & 64 & 7.40 & 0.47 & 5.47 & 0.66 & 0.62 \\
Controls & 15 & 5.40 & 0.43 & 5.28 & 0.68 & 0.64 \\
\hline
\end{tabular}

$n$ number of accessions, $A N$ average number of alleles per locus, MAF major allele frequency, $R_{S}$ average allelic richness. $H_{e}$, expected heterozygosity, PIC Polymorphic information content

had a low level of differentiation with $F_{s t}$ values ranging from 0.043 to 0.126 . Among the STRUCTURE groups, the greatest differentiation was observed between StrGr1 and 2 (0.290), and the lowest was between StrGr3 and $4(0.086)$.

\section{Genetic grouping in relation to reference rice accessions}

We sampled 150 diverse rice cultivars representing all five major groups identified in previous studies $[9,28]$ to analyze the genetic grouping of hill rice landraces included in this study. The reference dataset of 33 SSR markers (Additional file 4), common with the present study, was independently re-analysed to check the grouping of the accessions into five clusters as originally obtained by using a total of 169 SSRs [9]. Phylogenetic reconstruction confirmed a similar grouping of 150 reference rice accessions into five genetic clusters corresponding to indica, aus, aromatic, tropical japonica and temperate japonica (Additional file 9). In both NJ clustering and PCoA, the majority of the hill rice accessions were grouped in close association with aromatic and tropical japonica varieties (Fig. $5 \mathrm{a}$ and b, respectively). We also included a few japonica admixture varieties in the reference dataset to see their grouping in comparison to the hill rices. However, all the admixture varieties occupied a separate place in the genetic cluster. Difference in allelic richness was significant between hill and both tropical and temperate japonica (Fig. 5c). The pairwise estimates of $F_{s t}$ values based on allelic difference revealed that hill rice accessions had the highest degree of differentiation with temperate japonica (0.235), followed by the indica (0.231) (Additional file 8). The lowest $F_{s t}$ value was recorded between hill and admixture accessions (0.095).

\section{Discussion}

Our characterization of hill rice germplasm of NorthEastern region of India has revealed that a diverse array of rice landraces was grown by the farmers which traditionally classified as umte and tenning based on grain morphology and crop duration. These two groups of hill rice germplasm varied significantly for the traits such as plant height, leaf length, grain/kernel length and grain/ kernel length-to-width ratio, but non-significant genetic differentiation between the two groups were observed. Population genetic analysis showed that these hill rices were mostly japonica or admixture among the subpopulations of Indica or Japonica. These results are useful for understanding the agro-morphological variability, genetic diversity and structure of hill rice landraces, and significant for utilization of hill rices in crop improvement programmes. Our study also provides baseline information for planning in-situ conservation of the hill rice landraces, which are adapted to local environments in harmony with cultural preferences of the farmers.

\section{Hill rice landrace}

The traditional names of rice cultivars are generally found to be a valuable concept in describing the landraces worldwide. In the present study, the names of rice cultivars varied among the districts. Few rice accessions 'Taba,' 'Kilung' and 'Sarpung' were cultivated in all three districts. The farmers in Arunachal Pradesh most often name the rice landraces after their place of origin. For instance, 'Nepali dhan' is originated in Nepal. Likewise, 'Manipuri dhan' and 'Naga dhan' are from the state of Manipur and Nagaland, respectively. Rice landraces often named after a community/tribe. For example, 'Taba dugu' and 'Adi' named after 'Taba' and 'Adi' communities of Arunachal Pradesh, respectively. During collection trip the farmers expressed that hill rice cultivars generally taste better than the low land cultivars, and thus used for making local delicacies like pitha and sweets. Therefore, knowledge about the local names of rice landraces is useful for understanding their origin as well as grain quality and other characteristics. The traditional rice landraces possess wide genetic variability valuable for rice breeding [42, 43]. The broad genetic base of traditional rice cultivars is suitable for subsistence farming practiced by the farming communities. Rice germplasm collected from geographically isolated and ecologically important sites need to be characterized both at phenotypic and genetic levels to understand the interaction and genetic controls for traits influencing 


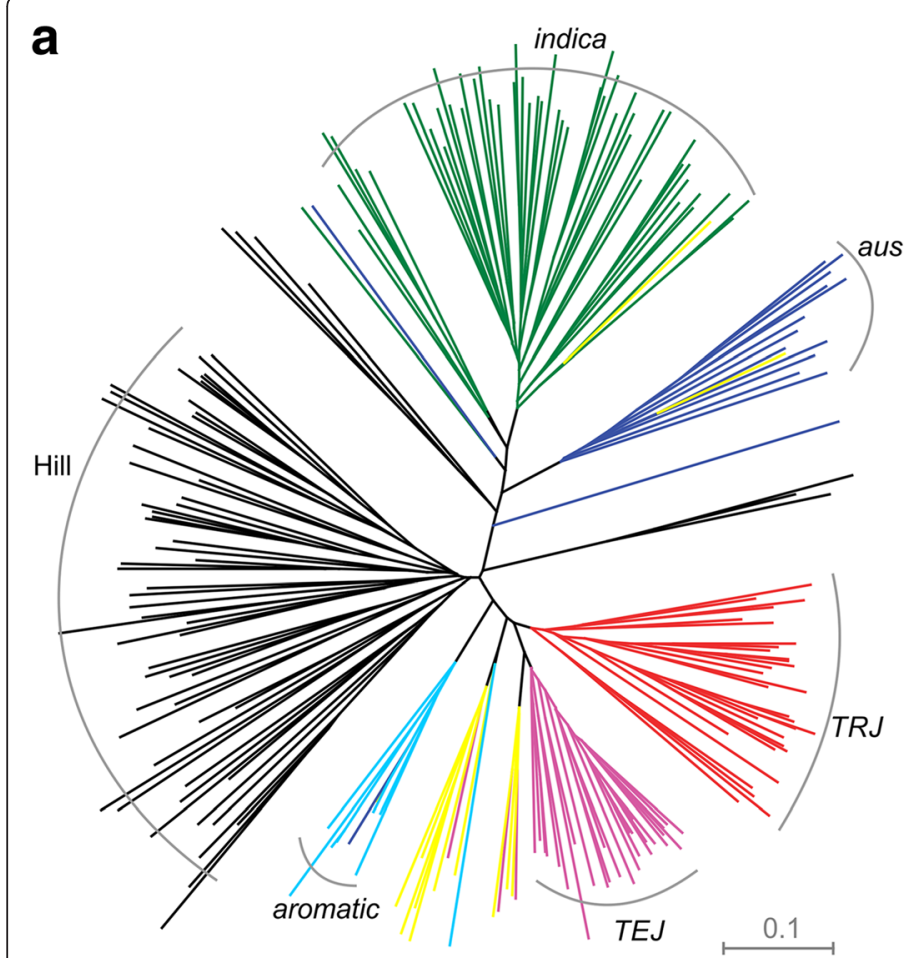

\section{b}

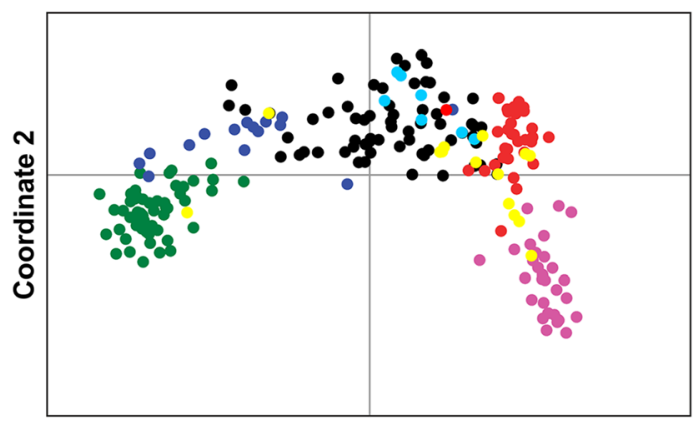

Coordinate 1

- Hill rice tropical japonica (TRJ)

- indica temperate japonica (TEJ)

- aus Admixture

- aromatic

C

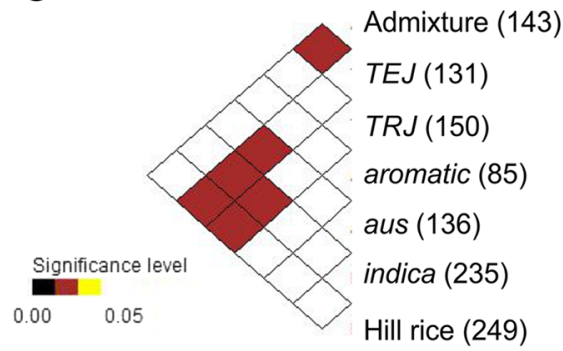

Fig. 5 Genetic grouping of 64 hill rice accessions in relation to the reference rice cultivars. a Neighbour-joining tree based on C. S. Chord distance; b Principal coordinate analysis; c Pair-wise tests of allelic differences between different groups of rice accessions. The number of observed alleles in each group is given in the parenthesis

adaptation of rice crop to harsh environments, and to identify sources for important adaptive traits [44]. In Nepal, the rice accessions sampled from mid- and highhill sites were found to possess tolerance to cold stress. However, interestingly rice diversity in the high-hill regions was lower than those of mid-hill and low-hill sites [45]. It was proposed that rice diversity in the mid-hills is linked with the range in altitude in these regions that results in great environmental heterogeneity and diverse agro-ecosystems, and great diversity in the socioeconomic structure of the farming communities [46].

\section{Agro-morphological differentiation}

The tribal farmers of Arunachal Pradesh usually classify hill rice cultivars on the basis of grain morphology and/ or quality characteristics, in addition to other agronomic traits such as crop duration. In the present study, we have assessed the variability in agronomic and grain/ kernel morphological traits to understand the morphological factors differentiating the two groups (umte and tening). Our analysis apparently showed that two groups of hill rice are mainly differentiated by the traits such as grain/kernel length and length-to-width ratio. We did not find significant variation in days to flowering between umte and tening. However, this is a key trait considered by the farmers in categorizing the hill rices. The non-significant variation in days to flowering in the present study may be due to change in crop growing conditions including soil and other environmental factors. We also performed a step-wise canonical discriminant analysis (CDA) taking umte and tening as priori groups to identify the most important discriminating traits contributing to the grouping of hill rices. Kernel length and plant height were found to be the best predictors for classifying the cultivar groups (results not shown). In both PCA and cluster analysis, we found that the accessions with similar names formed close groups, indicating the consistency in naming the cultivars by traditional farmers. However, in a different study with 130 indigenous rice accessions from a neighbouring state (Nagaland) in NE India, we found inconsistency in naming the cultivars [47]. Similar observation was also 
reported in upland rices of Nepal [48]. The grouping of hill rice accessions in both PCA and Ward's cluster did not follow geographical origin for a genotype. Similar trend was also recorded in previous studies [47, 49]. The average number of panicles per plant in hill rice accessions of Arunachal Pradesh (5.30) is lower than those reported in rice landraces from Nagaland state (7.9) [47] and rice cultivars adapted to high-altitude environments in Nepal (8.0) [48]. Extremely strong selection pressure for adaptation to the harsh high-altitude environments and direct seeding method of planting could have limited the number of productive tillers in hill rice germplasm.

\section{Genetic diversity and structure}

In the present study, we have used 35 SSR markers for genotyping rice landraces. A majority of these markers were chosen from the panel of 50 standard SSR markers recommended for rice diversity analysis by the Generation Challenge Programme by CGIAR. Among these SSRs, 33 markers also represent a subset of 169 markers originally used by Garris et al. [9]. Grouping 150 reference rice accessions from Garris et al. [9] using these 33 SSRs, was nearly the same. This suggests that the 35 SSR markers used in the present study can sufficiently resolve the genetic structure in rice.

Generally, the rice varieties grown under upland culture in the hill areas of Southeast Asia as a component of shifting cultivation are japonicas [1]. Earlier studies also indicated that a majority of upland rice landraces grown in upland fields are japonica [18]. However, upland indica cultivars were also recorded in different studies [20,50]. An earlier study targeting indica-japonica classification of Asian rice ecotypes and Japanese rice cultivars proposed that lowland rices can be clearly classified into indica or japonica, while, upland cultivars cannot [51]. In the present study, we attempted to classify the hill rice accessions by grouping them in relation to the control varieties. The STRUCTURE analysis revealed that considering the optimal grouping of the genotypes into two clusters, 79 rice accessions can be divided in to two broad groups: Indica and Japonica. Among the hill rices, only 18 accessions grouped with indica and aus control varieties, suggesting that the majority of hill rices are Japonica. Based on the STRUCTURE grouping at $K=4,50 \%$ of hill rice accessions were identified as japonica. The number of admixed individuals at $K=4$ was considerable higher than that at $K=2$, which suggests that the majority of hill rice cultivars are derived from admixture among the groups within either Indica or Japonica rather than between two subspecies. Similar findings were also reported in the Chinese rice collection [14]. Genetic grouping of rice accessions in both $\mathrm{NJ}$ cluster and PCoA also supported the STRUCTURE results. The genetic grouping of rice accessions in this study did not support the farmer's classification of hill rices. This discrepancy may be due to the limited number of SSR loci used in diversity analysis. These 35 SSR markers might reflect only a small part of the genotype-phenotype association of otherwise complex traits like grain and kernel length and shape, which are actually attributed to multiple loci with small effect $[52,53]$. Similar observations were also reported in upland rice accessions of Nepal when analysed with 36 SSR markers [48], and in a set of 91 rice accessions from Eastern and NE India using 23 SSR markers [54].

In the present study, the genetic grouping of hill rices based on SSR data was also inconsistent with the geographical distribution (district-wise) and naming of rice cultivars. We cannot explain why geographical distribution did not match with genetic relationship among accessions. Seed exchange between the villages might be the reason. We noted that the farmers in Arunachal Pradesh most often gift seeds of good rice varieties during wedding ceremonies. It has been observed that the landraces with the same name collected from different villages/district are not always genetically identical. Similar observations were also reported in other studies $[18,48]$. Therefore, during the germplasm collection, cultivars with the same names need to be collected as each accession is inherently valuable.

The current hill rice accessions revealed overall genetic diversity $\left(H_{e}=0.66\right.$; $\left.\mathrm{PIC}=0.62\right)$ similar to the 107 aromatic rice cultivars of $\mathrm{NE}$ India $\left(H_{e}=0.67\right.$; $\mathrm{PIC}=0.62)$ [30]. Slightly lower genetic diversity was reported in a subset of 26 rice accessions collected from NE India (PIC $=0.57$ ) [54]. The genetic diversity in the current hill rice accessions was considerably higher than that found in high-altitude rice cultivars of Nepal $(\mathrm{PIC}=0.17$ ) [48]. Although allelic diversity indices could be used as indicators of genetic variation in germplasm collection, such estimates are relative and largely depend on the number of polymorphic loci and relatedness of genotypes included [55]. The diverse nature of Arunachal Pradesh hill rices may be a reflection of the prevalent diverse agro-climatic, ethnocultural and eco-geographical features of the state. Interestingly, the genetic diversity between the umte and tening groups of hill rice was at par, and pair-wise allelic difference was non-significant. This is perhaps due to similar genetic makeup of the accessions of two morphological groups. The genetic diversity estimates among four STRUCTURE subpopulations were higher for group 3 and 4 ( $H_{e}=0.69$ and 0.59 , respectively), which can be categorized as japonica groups. However, the gene diversity in nine accessions of groups 2 was slightly lower $\left(H_{e}=0.53\right)$. This group was characterized by indica accessions, and 
higher genetic diversity of these accessions supports the fact that indica accessions are more diverse than japonica accessions $[15,56]$.

\section{Conclusions}

The present research, although with a limited number of SSR markers, indicated that hill rices were mostly japonica or admixture among the subpopulations of Indica or Japonica. Both FPTest and $F_{s t}$ results indicated that the hill accessions are genetically diverse from temperate japonica and indica. The rice germplasm prevailing in NE India has great importance in studying the evolution and population genetics of cultivated rice. This region is considered to be the domestication origin of Indica rices [1]. While, the adjoining Southern China is the proposed origin of Japonica rice $[1,57]$. Therefore, the bordering region of China and India can be considered as the differentiating zone of the two subspecies. Many studies in the past have reported the occurrence of diverse rice ecotypes, belonging to various genetic groups of rice, in NE India and Bangladesh [58]. Prevalence of Japonica rices has also been recorded in NE Indian rice germplasm [30, 59]. The hill rice germplasm reported in the present study may be a valuable resource for studying Indica-Japonica evolution.

The findings presented here also have some implications for the utilization and conservation of hill rice germplasm. First, considerable diversity, both in agro-morphological and genetic terms, has been maintained by the traditional farmers. This is most likely the result of maintaining different sets of landraces in different regions in response to local heterogeneities in climate and soil type, as well as indigenous culture, as demonstrated by Fu et al. [60] in Thai cassava farming. However, due to increased susceptibility to pests and diseases, some landraces have become obsolete. Second, our findings also are encouraging for onfarm conservation programme in the study location, along with the ex situ conservation efforts. The genetic variation among the cultivars from different districts was not significant, which indicates that on farm conservation programme can be initiated in any of the three districts of Arunachal Pradesh. Third, significant variations for many agro-morphological traits can be exploited in the rice breeding programmes. The information on genetic distance among the landraces could be used to guide the selection of valuable germplasm in rice breeding.

\section{Additional files}

Additional file 1: Hill rice cultivation in the state of Arunachal Pradesh a Jhum fields after rice harvesting; $b$ Harvesting of hill rice by a lady of Nyishi community in Papum Pare district; c rice granary containing harvested and stored grain; $d$ An overview of grain morphology of the hill rice accessions collected for the study. (PDF $2308 \mathrm{~kb}$ )
Additional file 2: Arunachal Pradesh villages where hill rice accessions were collected. (DOC $39 \mathrm{~kb}$ )

Additional file 3: Names, accession number, classification and genetic structure of 64 hill rice landraces and control varieties used in SSR fingerprinting. (DOC $135 \mathrm{~kb}$ )

Additional file 4: Details and summary statistics of 35 SSR markers used in the study. (DOC $73 \mathrm{~kb}$ )

Additional file 5: The original genotype data of hill rice accessions along with the control varieties. (XLSX $38 \mathrm{~kb}$ )

Additional file 6: Determination of the best $K$ value(s) based on mean $\operatorname{LnP}(D)$ over five runs for each $K$ value, and rate of change in the log probability of data between successive $K$ values $(\Delta K)$. (PDF $1363 \mathrm{~kb}$ )

Additional file 7: Analysis of SSR molecular variance. (DOC $32 \mathrm{~kb}$ ) Additional file 8: Pair-wise $F_{s t}$ values for different sub-populations. (DOC $58 \mathrm{~kb}$ )

Additional file 9: Phylogenetic reconstruction of 150 reference rice accessions (Garris et al., 2005) based on 33 SSR markers data. (PDF 1358 kb)

\section{Abbreviations}

AMOVA, analysis of molecular variance; AN, average number of alleles per locus; ANOVA, analysis of variance; CDA, canonical discriminant analysis; CGIAR, Consultative Group on International Agricultural Research; CV, coefficient of variation; ICAR, Indian Council of Agricultural research; MAF, major allele frequency; NJ, neighbour joining; PCA, principal component analysis; PCoA, principal coordinate analysis; PCR, polymerase chain reaction; PIC, polymorphism information content; SD, standard deviation; SSR, Simple sequence repeats

\section{Acknowledgement}

The work was supported by Indian Council of Agricultural Research under the project IXX04376. The exploration trip for collecting hill rice landraces from Arunachal Pradesh, India was undertaken as per approved exploration programme of ICAR-National Bureau of Plant Genetic Resources, New Delhi, India. The authors wish to thank Dr SV Ngachan, Director, ICAR Research Complex for NEH Region for providing laboratory facilities to carry out the SSR genotyping. They also wish to thank Dr Trilochan Mohapatra, former Director, ICAR-National Rice Research Institute, Cuttack, Odisha for providing the control varieties of rice. The authors are thankful to Dr Mo-Hua Yang, Associate Professor, Central South University of Forestry and Technology, China for performing the FPTest analysis in R.

\section{Availability of data and materials}

The data sets supporting the results of this article are included within the article and its additional files.

\section{Authors' contributions}

$\mathrm{SR}, \mathrm{BCM}$ conducted the exploration trip to Arunachal Pradesh for hill rice collection. SR conceived the experiment. SR, AB designed the experiment. $B M, S R, A B, R M Y$ conducted all the experiments pertaining to DNA extraction, PCR and SSR genotyping. SR, RMY conducted field experiments and phenotyping of rice accessions. SR conducted the data analysis and drafted the manuscript. KCB, AB, AKM revised the manuscript. All authors have read and approved the final manuscript.

\section{Competing interests}

The authors declare that they have no competing interests.

Consent for publication

Not applicable.

Ethics approval and consent to participate Not applicable.

\section{Author details}

'ICAR-National Bureau of Plant Genetic Resources, Regional Station, Umiam, Meghalaya 793103, India. ${ }^{2}$ ICAR-National Rice Research Institute, Cuttack, 
Odisha 753006, India. ${ }^{3}$ ICAR Research Complex for NEH Region, Umiam, Meghalaya 793103, India. ${ }^{4}$ ICAR-National Bureau of Plant Genetic Resources, New Delhi 110012, India. ${ }^{5}$ Present address: ICAR-National Rice Research Institute, Cuttack, Odisha 753006, India.

\section{Received: 9 February 2016 Accepted: 7 July 2016 Published online: 13 July 2016}

\section{References}

1. Khush GS. Origin, dispersal, cultivation and variation of rice. Plant Mol Biol. 1997;35:25-34.

2. Wing RA, Ammiraju JSS, Luo MZX, Kim H, Yu YS, Kudrna D, et al. The Oryza Map Alignment Project: the golden path to unlocking the genetic potential of wild rice species. Plant Mol Biol. 2005;59:53-62.

3. Zhang QF, Li JY, Xue YB, Han B, Deng XW. Rice 2020: a call for an international coordinated effort in rice functional genomics. Mol Plant. 2008;1:715-9.

4. Oka HI. Origin of Cultivated Rice. Tokyo: Japan Scientific Societies Press; 1988.

5. Sang T, Ge S. Genetics and phylogenetics of rice domestication. Curr Opin Genet Dev. 2007;17:533-8.

6. Sweeney M, McCouch S. The complex history of the domestication of rice Ann Bot. 2007;100:951-7

7. Glaszmann JC. Isozymes and classification of Asian rice varieties. Theor Appl Genet. 1987;74:21-30.

8. $\mathrm{Ni}$ J, Colowit PM, Mackill DJ. Evaluation of genetic diversity in rice subspecies using microsatellite markers. Crop Sci. 2002;42:601-7.

9. Garris AJ, Tai TH, Coburn J, Kresovich S, McCouch S. Genetic structure and diversity in Oryza sativa L. Genetics. 2005;169:1631-8.

10. Caicedo AL, Williamson SH, Hernandez RD, Boyko A, Fledel-Alon A, York TL, et al. Genome-wide patterns of nucleotide polymorphism in domesticated rice. PLoS Genet. 2007:3:1745-56.

11. Ebana K, Yonemaru J, Fukuoka S, Iwata H, Kanamori H, Namiki N, et al. Genetic structure revealed by a whole-genome single-nucleotide polymorphism survey of diverse accessions of cultivated Asian rice (Oryza sativa L.). Breeding Sci. 2010;60:390-7.

12. Zhao KY, Wright M, Kimball J, Eizenga G, McClung A, Kovach M, et al. Genomic diversity and introgression in O. sativa reveal the impact of domestication and breeding on the rice genome. PLoS ONE. 2010;5:e10780.

13. Huang XH, Zhao Y, Wei XH, Li CY, Wang A, Zhao Q, et al. Genome-wide association study of flowering time and grain yield traits in a worldwide collection of rice germplasm. Nat Genet. 2012;44:32-53.

14. Wang $\mathrm{C}-\mathrm{H}$, Zheng X-M, Xu Q, Yuan X-P, Huang L, Zhou H-F, et al. Genetic diversity and classification of Oryza sativa with emphasis on Chinese rice germplasm. Heredity. 2014;112:489-96.

15. Gao LZ, ChiHong Z, LiPing C, JiZeng J, ZongEn Q, Dong YS. Microsatellite diversity within Oryza sativa with emphasis on indica-japonica divergence. Genet Res. 2005:85:1-14

16. Prashanth SR, Parani M, Mohanty BP, Talame V, Tuberosa R, Parida A. Genetic diversity in cultivars and landraces of Oryza sativa subsp. indica as revealed by AFLP markers. Genome. 2002;45:451-9.

17. Barry MB, Pham JL, Noyer JL, Courtois B, Billot C, Ahmadi N. Implications for in situ genetic resource conservation from the ecogeographical distribution of rice genetic diversity in Maritime Guinea. Plant Genet Resour. 2007;5:45-54.

18. Thomson MJ, Polato NR, Prasetiyono J, Trijatmiko KR, Silitonga TS, McCouch SR. Genetic diversity of isolated populations of Indonesian landraces of rice (Oryza sativa L.) collected in East Kalimantan on the island of Borneo. Rice. 2009;2:80-92.

19. de Oliveira Borba TC, Brondani RP, Rangel PH, Brondani C. Microsatellite marker mediated analysis of the EMBRAPA Rice Core Collection genetic diversity. Genetica. 2009;137:293-304.

20. Zhang H, Sun J, Wang M, Liao D, Zeng Y, Shen S, et al. Genetic structure and phylogeography of rice landraces in Yunnan, China, revealed by SSR. Genome. 2007:50:72-83.

21. Zhang DL, Zhang HL, Wang MX, Sun JL, Qi YW, Wang F, et al. Genetic structure and differentiation of Oryza sativa $\mathrm{L}$. in China revealed by microsatellites. Theor Appl Genet. 2009:119:1105-17.

22. Li X, Yan W, Agrama H, Hu B, Jia L, Jia M, et al. Genotypic and phenotypic characterization of genetic differentiation and diversity in the USDA rice mini-core collection. Genetica. 2010;138:1221-30.

23. Vaughan DA, Lu BR, Tomooka N. The evolving story of rice evolution. Plant Sci. 2008;174:394-408.
24. Hore DK. Rice diversity collection, conservation and management in Northeastern India. Genet Resour Crop Evol. 2005;52:1129-40.

25. Government of India. Basic statistics of North Eastern region 2015 Government of India, North Eastern Council Secretariat, Nongrim Hills, Shillong, India; 2015.p.411

26. Directorate of Rice Development (2015) State-wise rice productivity analysis, State: Arunachal Pradesh. Directorate of rice development, Department of Agriculture and Co-operation, Patna, Bihar; http://drdpat.bih.nic.in. Accessed 30 December, 2015.

27. Sthapit BR. Chilling injury of rice crop in Nepal: A review. J Inst Agric Anim Sci. 1992:13:1-32

28. McCouch SR, Wright MH, Tung C-W, Maron LG, McNally KL, Fitzgerald M, et al. Open access resources for genome-wide association mapping in rice. Nat Commun. 2016;7:10532.

29. IBPGR-IRRI. Descriptors for rice (Oryza sativa L.) IBPGR-IRRI Rice Advisory Committee. Manila: IRRl; 1980.

30. Roy S, Banerjee A, Mawkhlieng B, Misra AK, Pattanayak A, Harish GD, et al, Genetic diversity and population structure in aromatic and quality rice (Oryza sativa L.) landraces from North-Eastern India. PLoS ONE. 2015;10:e0129607.

31. IBM Corp. IBM SPSS Statistics for Windows, version 20.0. Armonk: IBM Corp; 2011

32. Pritchard JK, Stephens M, Donnelly P. Inference of population structure using multilocus genotype data. Genetics. 2000;155:945-59.

33. Evanno G, Regnaut S, Goudet J. Detecting the number of clusters of individuals using the software STRUCTURE: a simulation study. Mol Ecol. 2005;14:2611-20.

34. Liu KJ, Muse SV. PowerMarker: an integrated analysis environment for genetic marker analysis. Bioinformatics. 2005;21:2128-9.

35. Goudet J. FSTAT version 1.2: a computer program to calculate F statistics. J Hered. 1995:86:485-6.

36. Peakall R, Smouse PE. GenAlEx 6.5: genetic analysis in Excel. Population genetic software for teaching and research-an update. Bioinformatics. 2012;28:2537-9.

37. R Core Team. R: A language and environment for statistical computing. Vienna: R Foundation for Statistical Computing; 2016.

38. Fu YB, Peterson GW, Scoles G, Rossnagel B, Schoen DJ, Richards KW. Allelic diversity changes in 96 Canadian oat cultivars released from 1886 to 2001. Crop Sci. 2003;43:1989-95.

39. Rohlf F. NTSYS-pc: Numerical Taxonomy and Multivariate Analysis System, Version 2.00. Setauket: Exeter Software; 1997.

40. Cavalli-Sforza LL, Edwards AWF. Phylogentic analysis. Models and estimation procedures. Am J Hum Genet. 1967;19:233-57.

41. Huson DH, Scornavacca C. Dendroscope 3 - An interactive viewer for rooted phylogenetic trees and networks. Syst Biol. 2012;61(6):1061-7.

42. Fukuoka S, Suu T, Ebana K, Trinh L, Nagamine T, Okuno K. Diversity in phenotypic profiles in landrace populations of Vietnamese rice: a case study of agronomic characters for conserving crop genetic diversity on farm. Genet Resour Crop Evol. 2006:53:753-61.

43. Rana RB, Garforth C, Sthapit B, Jarvis D. Influence of socio-economic and cultural factors in rice varietal diversity management on-farm in Nepal. Agric Human Values. 2007;24:461-72.

44. Sharma RC, Chaudhary NK, Ojha B, Yadav L, Pandey MP, Shrestha SM Variation in rice landraces adapted to the lowlands and hills in Nepal. Plant Genet Resour: Charact Util. 2007:5:120-7.

45. Bajracharya J, Rana RB, Gauchan D, Sthapit BR, Jarvis DI, Witcombe JR. Rice landrace diversity in Nepal. Socio-economic and ecological factors determining rice landrace diversity in three agro-ecozones of Nepal based on farm surveys. Genet Resour Crop Evol. 2010;57:1013-22.

46. Sthapit BR, Joshi KD, Rana RB, Upadhayay MP, Eyzaguirre P, Jarvis D. Enhancing biodiversity and production through participatory plant breeding: setting breeding goals. In: An exchange of experiences from South and South East Asia. Proceedings of the International Symposium on Participatory Plant Genetic Resource Enhancement. 2000.

47. Roy S, Rathi RS, Misra AK, Bhatt BP, Bhandari DC. Phenotypic characterization of indigenous rice (Oryza sativa L.) germplasm collected from the state of Nagaland. Plant Genet Resour: Charact Util. 2014;12:58-66.

48. Bajracharya J, Steele KA, Jarvis DI, Sthapit BR, Witcombe JR. Rice landrace diversity in Nepal: variability of agro-morphological traits and SSR markers in landraces from a high-altitude site. Field Crop Res. 2006:95(2-3):327-35.

49. Mathure S, Shaikh A, Renuka N, Wakte K, Jawali N, Thengane R, Nadaf A Characterisation of aromatic rice (Oryza sativa L.) germplasm and correlation between their agronomic and quality traits. Euphytica. 2011;179:237-46. 
50. Pessoa-Filho M, Beló A, Alcochete A, Rangel P, Ferreira M. A set of multiplex panels of microsatellite markers for rapid molecular characterization of rice accessions. BMC Plant Biol. 2007;7:23.

51. Ueno K, Sato T, Takahashi N. The indica-japonica classification of Asian rice ecotypes and Japanese lowland and upland rice (Oryza sativa L.). Euphytica. 1990:46:161-4

52. Ge XJ, Xing YZ, Xu CG, He YQ. QTL analysis of cooked rice grain elongation, volume expansion, and water absorption using a recombinant inbred population. Plant Breed. 2005;124:121-6.

53. Bai X, Luo L, Yan W, Rao MK, Zhan W, Xing Y. Genetic dissection of rice grain shape using a recombinant inbred line population derived from two contrasting parents and fine mapping a pleiotropic quantitative trait locus qGL7. BMC Genet. 2010;11:16.

54. Das B, Sengupta S, Parida SK, Roy B, Ghosh M, Prasad M, et al. Genetic diversity and population structure of rice landraces from Eastern and North Eastern states of India. BMC Genet. 2013;14:71.

55. Ren $X$, Jiang $H$, Yan Z, Chen $Y$, Zhou X, Huang L, et al. Genetic diversity and population structure of the major peanut (Arachis hypogaea L.) cultivars grown in China by SSR markers. PLoS ONE. 2014;9:e88091.

56. Ali ML, McClung AM, Jia MH, Kimball JA, McCouch SR, Eizenga GC. A rice diversity panel evaluated for genetic and agro-morphological diversity between subpopulations and its geographic distribution. Crop Sci. 2011;51:2021-35.

57. Huang X, Kurata N, Wei X, Wang Z-X, Wang A, Zhao Q, et al. A map of rice genome variation reveals the origin of cultivated rice. Nature. 2012:490:497-501

58. Travis AJ, Norton GJ, Datta S, Sarma R, Dasgupta T, Savio FL, et al. Assessing the genetic diversity of rice originating from Bangladesh, Assam and West Bengal. Rice. 2015;8:35

59. Choudhury B, Khan ML, Dayanandan S. Genetic structure and diversity of indigenous rice varieties (Oryza sativa) in Eastern Himalayan region of Northeast India. Springer Plus. 2013;2:228-37.

60. Fu YB, Wangsomnuk PP, Ruttawa B. Thai elite cassava genetic diversity was fortuitously conserved through farming with different sets of varieties. Conserv Genet. 2014;15:1463-78.

\section{Submit your next manuscript to BioMed Central and we will help you at every step:}

- We accept pre-submission inquiries

- Our selector tool helps you to find the most relevant journal

- We provide round the clock customer support

- Convenient online submission

- Thorough peer review

- Inclusion in PubMed and all major indexing services

- Maximum visibility for your research

Submit your manuscript at www.biomedcentral.com/submit 